\title{
COLONIC DIVERTICULAR DISEASE
}

nature reviews disease primers

Colonic diverticular disease
(referred to as diverticular disease) - characterized by sac-like protrusions (diverticula) on the wall of the large intestine - can be asymptomatic

(known as diverticulosis) or

symptomatic (such as diverticulitis or diverticular haemorrhage).

\section{EPIDEMIOLOGY}

The prevalence of diverticulosis is increasing globally. In western countries, nearly two-thirds of adults are estimated to develop diverticulosis in their lifetime and $\sim 25 \%$ of these individuals develop diverticular disease. The prevalence of diverticulosis is very low in individuals of $<40$ years of age and increases with age. Approximately

$2-3 \%$ of individuals with diverticulosis develop diverticulitis and $<5 \%$ of individuals with diverticulosis experience diverticular haemorrhage.

Risk factors include age, genetic predisposition, lifestyle, diet and medication such as asprins, NSAIDs and corticosteroids.

\section{DIAGNOSIS}

Imaging evaluations such as $\mathrm{CT}$,

ultrasonography and MRI are mandatory for an accurate diagnosis of diverticular disease. Once diagnosed, C-reactive protein is the most useful biomarker to determine disease severity. Traditionally, colonoscopy was avoided in patients with acute diverticulitis owing to risks of perforation from the procedure, although the procedure is recommended before surgery or after an episode of acute diverticulitis to rule out or screen for colorectal cancer.

combination of increased colonic pressure, abnormal colonic motility and weakness of the colon wall is attributed to the

development of a diverticulum

\section{MECHANISMS}

Bacterial

stasis

stasis
eventually leads

micro-perforation,

inflammation and infection; in severe fistulas can form
(1)

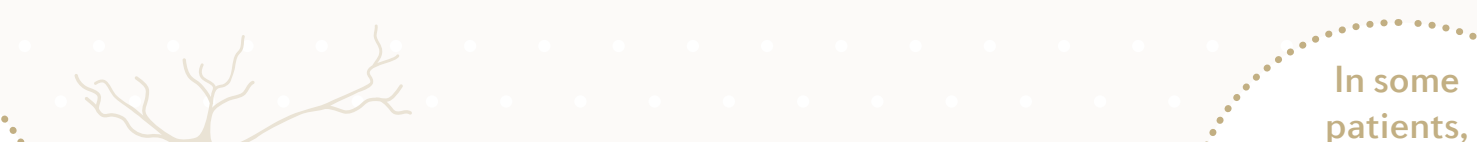
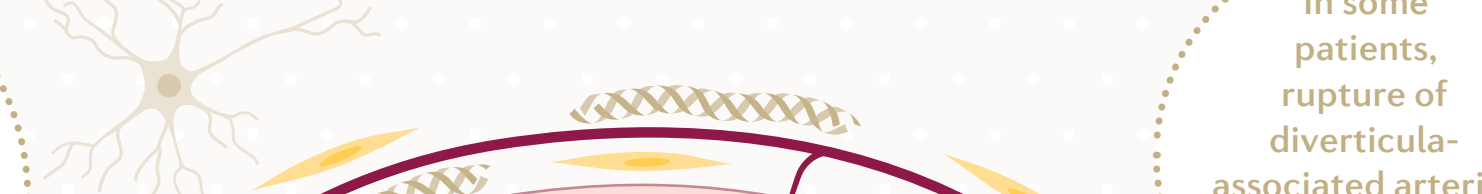

diverticula-

associated arteries

leads to colonic bleeding

.

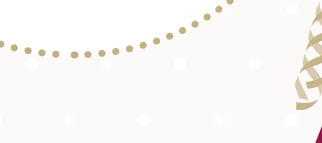

characterized by

inflammation mediated

by neuropeptides, which

might trigger visceral

some patients

some patients

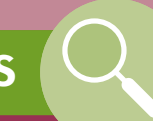
-
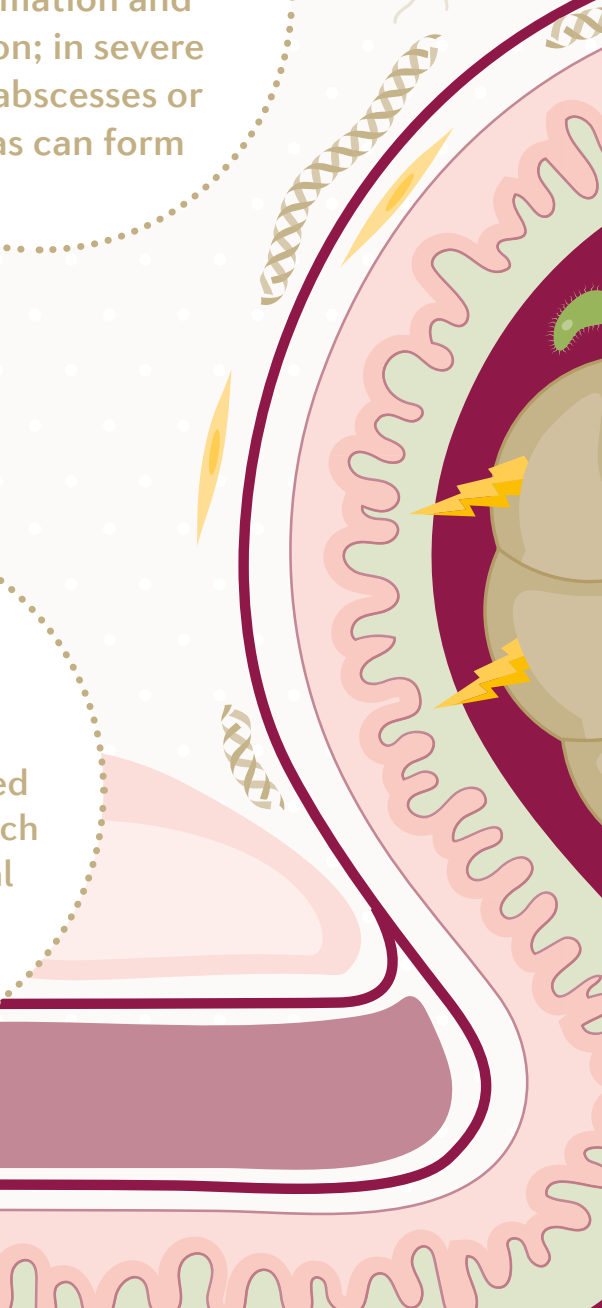

\section{QUALITY OF LIFE}

Patients often report recurrent abdominal pain, changes in bowel habits and bloating. The diverticulitis quality of life (DV-QOL) tool, a disease-targeted questionnaire, can be used to monitor the physical, psychological and social well-being of patients.
For the Primer, visit doi:10.1038/541572-020-0153-5

\section{MANAGEMENT}

Management of diverticular disease depends on the severity of the disease. Pharmacological therapy is not necessary for individuals with diverticulosis as most will not progress to symptomatic disease. For patients with symptoms, such as mild to moderate pain and bloating, a high-fibre diet is usually recommended. Poorly absorbed antibiotics (such as rifaximin) are prescribed to treat dysbiosis and to reduce gasrelated symptoms. Some studies report that anti-inflammatory drugs such as mesalazine can also relieve symptoms. For patients with 'uncomplicated' diverticulitis (that is, without abscess, fistula or perforation), outpatient management with antimicrobial therapy is used. However, owing to the evolving concepts in the pathogenesis (that the disease is an inflammatory process rather than an infection), the use of antibiotics in the treatment is disputed. For patients who present with large abscesses, percutaneous drainage is performed when antibiotic therapy alone is insufficient. However, $10-20 \%$ of patients (with sepsis or peritonitis) will eventually require surgery to relieve symptoms.

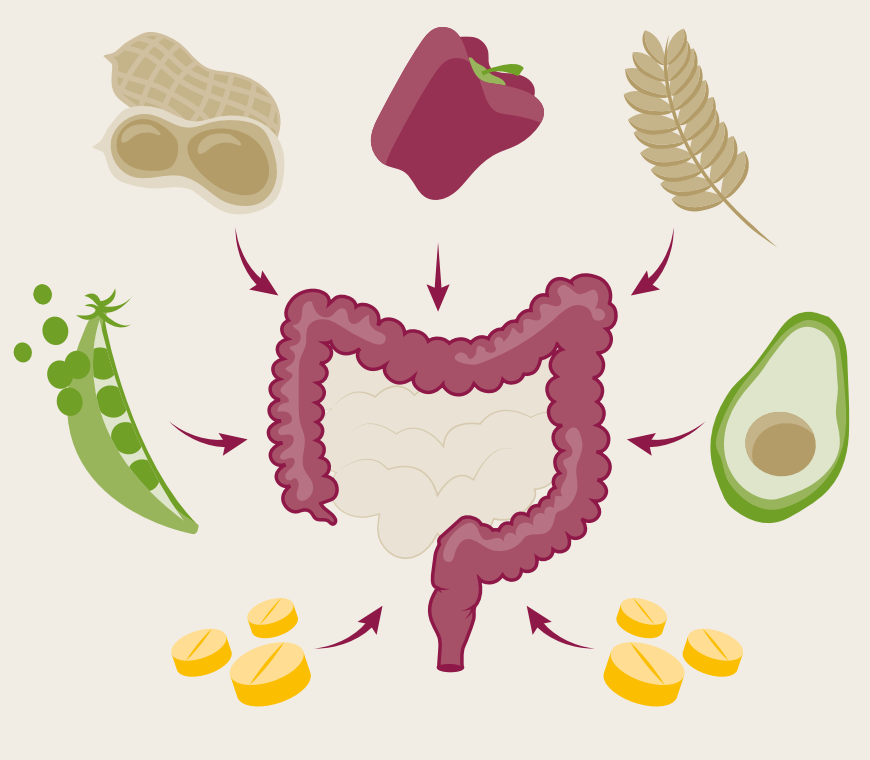

